\title{
Pediatric Acute Kidney Injury: Different From Acute Renal Failure, But How And Why?
}

\author{
Prasad Devarajan
}

Published online: 22 December 2012

(C) Springer Science + Business Media New York 2012

\begin{abstract}
Acute kidney injury [AKI] refers to a clinical syndrome encompassing various etiologies and occurring in a variety of clinical settings, with manifestations ranging from subtle biochemical and structural changes, to minimal elevation in serum creatinine, to anuric renal failure. Understanding the spectrum of AKI and the importance of the early pre-clinical damage stage has resulted in an improved ability to define and stage pediatric AKI, to understand the AKI-to-CKD transition, and harness novel damage biomarkers to predict AKI and its adverse outcomes. These concepts are expanded upon in this review, with an emphasis on publications from the past three years.
\end{abstract}

Keywords Acute kidney injury - Acute renal failure . Biomarkers · Neutrophil gelatinase-associated liopcalin

\section{Introduction}

For several decades, clinicians have used the term acute renal failure $[\mathrm{ARF}]$ to designate the discrete event of a failed kidney, characterized by a rapid accumulation of blood urea nitrogen and creatinine. However, ARF over-emphasizes the failure of kidney function and does not account for the diverse molecular, biochemical, and structural processes that transpire in an acutely injured kidney well before the decline in function. Leading thinkers have therefore proposed the term "acute kidney injury" (AKI). This refers to a

\footnotetext{
P. Devarajan ( $\square)$

Center for Acute Care Nephrology, Division of Nephrology and Hypertension, Cincinnati Children's Hospital Medical Center, University of Cincinnati School of Medicine, MLC 7022, 3333 Burnet Avenue, Cincinnati, OH 45229-3039, USA

e-mail: prasad.devarajan@cchmc.org
}

broad clinical syndrome encompassing various etiologies and occurring in a variety of clinical settings, with manifestations ranging from subtle biochemical and structural changes, to minimal elevation in serum creatinine, to anuric renal failure $\left[1^{\bullet \bullet}\right]$. The conceptual model for AKI starts with subjects who are at increased risk (due to genetic or clinical risk factors), and proceeds through an intermediate and previously unrecognized damage stage (now identified by novel biomarkers) to the stage of functional ARF [1••, 2]. Embracing the spectrum of AKI and the importance of the early pre-clinical damage stage has resulted in several paradigm-shifting outcomes. First, there has been an improvement in our ability to define, classify, and stage pediatric AKI [1••, 3]. Second, we now know that even very small early increases in serum creatinine predict the subsequent development of overt clinical AKI in children [4•]. Third, we have witnessed a revolution in the early prediction of AKI and its outcomes using novel damage biomarkers pioneered largely in pediatric studies [5]. Fourth, we have come to realize that pediatric AKI is plagued with common and serious adverse outcomes [6-8]. These concepts are expanded upon in the subsequent sections of this review, with an emphasis on publications from the past three years.

\section{Pediatric AKI Should be Defined in a Standardized Manner}

Historically, progress in pediatric AKI was hindered by the myriad definitions. During the past decade, two major classification systems have emerged (RIFLE and AKIN), based on serum creatinine and urine output criteria. A modification of the RIFLE criteria was suggested for pediatric use ( $\mathrm{pRIFLE),} \mathrm{substituting} \mathrm{serum} \mathrm{creatinine} \mathrm{val-}$ ues with estimated creatinine clearance [using the Schwartz 
formula]. Recent pediatric AKI studies have employed the pRIFLE criteria to report on AKI incidence, severity of illness, length of hospital stay, and mortality [4・, 9-11]. However, a systematic review of 12 pediatric studies using RIFLE or pRIFLE classification reported wide variations in the application of the criteria, and inconsistencies in the relationships between the RIFLE class and measures of morbidity and mortality [12]. Consequently, the precise incidence, prevalence, and outcomes of pediatric AKI still remain unclear.

Recognizing the need for a single consensus definition and staging system that could be applied to both children and adults, the Kidney Disease: Improving Global Outcomes (KDIGO) group has proposed the following definition for AKI $[1 \bullet \cdot]$ :

- Increase in serum creatinine by $\geq 0.3 \mathrm{mg} / \mathrm{dl}$ [ $\geq 26.5 \mu \mathrm{mol} / \mathrm{l}]$ within $48 \mathrm{~h}$; OR.

- Increase in serum creatinine to $\geq 1.5$ times baseline within the prior 7 days; OR.

- Urine volume $<0.5 \mathrm{ml} / \mathrm{kg} / \mathrm{h}$ for $6 \mathrm{~h}$.

The KDIGO staging of AKI is illustrated in Table 1, and incorporates RIFLE, pRIFLE, and AKIN classifications. Both the definition and staging feature a $0.3 \mathrm{mg} / \mathrm{dl}$ increase in serum creatinine to specifically be applicable to pediatric AKI. The KDIGO staging also allows for a child with eGFR $<35 \mathrm{ml} / \mathrm{min}$ per $1.73 \mathrm{~m}^{2}$ to be classified as Stage 3, in contrast with the adult criterion of $\geq 4 \mathrm{mg} / \mathrm{dl}$ serum creatinine (which would be unrealistic in infants and young children). The uniform adoption of the KDIGO definition and staging of AKI holds significant promise for improving our understanding of pediatric AKI epidemiology, and therefore deserves our undivided attention.

\section{Pediatric AKI is Common but Lacks Awareness}

There is now growing evidence to indicate that pediatric AKI is not only common, but also rising in incidence. Potential explanations for the mounting incidence rate include the increased availability of treatment options for many critical illnesses (sepsis, congenital heart disease, bone marrow transplants), and advances in neonatal and pediatric intensive care. While pediatric centers have previously reported an AKI incidence of only $1 \%$ of all general hospital admissions, the incidence is substantially higher in specialized populations with critical illnesses that are now routinely managed. Recent retrospective and prospective studies from around the globe indicate an AKI incidence of $10-35 \%$ (by RIFLE or AKIN criteria) among children admitted to pediatric intensive care units [13••, 14 , 15]. This incidence jumps up to nearly $90 \%$ if only mechanically ventilated children with trauma or vasopressor requirement are included [16]. In children undergoing cardiac surgery, reports from several countries indicate an AKI incidence (by RIFLE or AKIN criteria) of $30-50 \%$ [17-22]. Even among non critically ill children, the incidence of AKI by pRIFLE criteria was high at $34 \%$ when those receiving potentially nephrotoxic medications were analyzed [23]. Thus, pediatric AKI is reaching epidemic proportions, especially among critically ill children, in whom kidney failure is just as common as other major organ failures. Indeed, among adults, AKI has a general incidence of 2.1/1000 population, similar to that of acute myocardial infarction, the latter condition clearly much more widely appreciated by clinicians, researchers, and the general public [24]. Future studies employing consensus definitions in a rigorous manner will be necessary to raise awareness of this increasingly common condition.

\section{Pediatric AKI Epidemiology has Changed}

The epidemiology and etiology of pediatric AKI is greatly influenced by the clinical setting and geographic location. In developed countries, the epidemiology of pediatric AKI has shifted during the past two decades from primary glomerular diseases to a hospital-acquired complication of other systemic illnesses. The most prevalent causes now include sepsis, congenital heart disease, ischemic injury in critically ill neonates and children, nephrotoxins, and malignancy $[9,10]$. Even in underdeveloped countries, the pattern of pediatric AKI in urban areas closely resembles that seen in the more developed countries [10]. Ironically, even tragically, pediatric AKI has become the consequence of improved critical care.
Table 1 KDIGO staging of AKI

Adapted from Reference 1

\begin{tabular}{lll}
\hline Stage & Serum creatinine & Urine output \\
\hline 1 & $1.5-1.9$ times baseline, OR $\geq 0.3 \mathrm{mg} / \mathrm{dl}(\geq 26.5 \mu \mathrm{mol} / \mathrm{l})$ increase & $<0.5 \mathrm{ml} / \mathrm{kg} / \mathrm{h}$ for $6-12 \mathrm{~h}$ \\
2 & $1.0-2.9$ times baseline & $<0.5 \mathrm{ml} / \mathrm{kg} / \mathrm{h} \mathrm{for} \geq 12 \mathrm{~h}$ \\
3 & $\begin{array}{c}3.0 \text { times baseline, OR } \mathrm{SCr} \geq 4.0 \mathrm{mg} / \mathrm{dl}(\geq 353.6 \mu \mathrm{mol} / \mathrm{l}), \mathrm{OR} \\
\text { Initiation of renal replacement therapy, OR eGFR }<35 \mathrm{ml} / \mathrm{min}\end{array}$ & $\begin{array}{c}<0.3 \mathrm{ml} / \mathrm{kg} / \mathrm{h} \mathrm{for} \geq 24 \mathrm{~h}, \mathrm{OR} \\
\text { Anuria for } \geq 12 \mathrm{~h}\end{array}$ \\
& per $1.73 \mathrm{~m}^{2}(<18$ years $)$ & \\
\hline
\end{tabular}




\section{Pediatric AKI has Important Consequences}

The short-term outcomes of AKI have been well documented in children undergoing cardiac surgery. In a prospective multinational study of 311 children undergoing cardiac surgery, AKI was independently associated with prolonged mechanical ventilation and increased length of hospital stay [19]. This independent association has now been confirmed in other large studies from several countries [20-22]. Importantly, a retrospective analysis of infants who developed AKI after cardiac surgery revealed a mortality rate of $7 \%$; multivariable logistic regression analysis showed that more severe AKI was associated with greater in-hospital mortality [20]. For AKIN stage II, the odds ratio of death was 5.1 and for AKIN stage III, it was 9.46 .

The short-term outcomes of children with AKI as a complication of critical illness are also well known. In a multicenter retrospective analysis of 2,106 pediatric ICU admissions, AKI was independently associated with longer ICU stay and mechanical ventilation [14]. A recent large retrospective study of 3,396 admissions to a single pediatric ICU illustrated that those who presented with AKI on admission had a $32 \%$ mortality rate and those who developed AKI at any time during the ICU stay had a $30 \%$ mortality rate [13*0]. Remarkably, this persistently high mortality rate of 30-40\% in critically ill children with AKI has been consistently demonstrated in several very recent studies [9, 10, 14-16, 25].

Thus, notwithstanding advances in pediatric renal and critical care, severe AKI requiring renal replacement therapy in children is still associated with a mortality rate of 30-50\%, and this has not changed appreciably over the past two decades. This may reflect, in part, the fact that those with severe AKI also have increasing severity of their primary illness, so that an improvement in survival rates is not readily apparent despite renal replacement therapy. This notion is supported by a multicenter retrospective analysis of 344 children requiring continuous renal replacement therapy, in whom the overall mortality rate was $42 \%$ [26]. However, there was significantly better survival in patients with less severity of their primary illness, including drug intoxication (100\%), primary renal disease $(84 \%)$, and tumor lysis syndrome (83\%). Despite dialysis, survival was lowest in the sickest children with liver disease $(43 \%)$, pulmonary disease (45\%), and bone marrow transplant $(45 \%)$.

The previous assumption that patients who survived an episode of AKI would recover kidney function has been challenged. A recent meta-analysis of 13 published cohort studies showed that adults with AKI are at a ninefold higher risk of developing CKD, and a threefold increased risk of developing ESRD, when compared to patients without AKI [27]. Similar evidence is accumulating in the pediatric population $[6-8,28]$. In a prospective study of children who developed AKI and were followed up for 3-5 years, $60 \%$ developed evidence for CKD (proteinuria, decreased GFR, hypertension), $9 \%$ developed ESRD, and $20 \%$ died [29]. A shorter 1-3 year follow up of 126 critically ill children who suffered an episode of AKI [30•] showed that $10 \%$ developed CKD (eGFR $<60 \mathrm{ml} / \mathrm{min}$ per $1.73 \mathrm{~m}^{2}$ or albuminuria), but $47 \%$ showed evidence of CKD risk (eGFR $60-90 \mathrm{ml} / \mathrm{min}$ per $1.73 \mathrm{~m}^{2}$ or hypertension). However, several unknowns remain. Can clinical risk factors (e.g., etiology and severity of AKI, pre-existing $\mathrm{CKD}$, other co-morbid factors) determine the risk of CKD after AKI? Can novel biomarkers predict this risk? Can early interventions prevent progression of CKD? Ongoing long-term follow up studies in both children and adults [31] are expected to clarify some of these questions, but clearly a lot more work is needed to strengthen the emerging recommendation that children with AKI require long-term evaluation for CKD. Future studies should also incorporate lessons learned from contemporary basic science studies that have unveiled the mechanisms leading to vascular rarefaction and progressive interstitial fibrosis after AKI [32], and the role of novel regulatory proteins such as neutrophil gelatinase-associated lipocalin and kidney injury molecule-1 as pathogenic factors as well as early biomarkers for the AKI-to-CKD transition [33].

\section{The Causes of Death in Pediatric AKI Have Changed}

Modern renal replacement therapies have largely eliminated the traditional life-threatening complications of AKI, including hyperkalemia, arrhythmias, and uremic coma. Why, then, do children with AKI still die? Both clinical and experimental evidence points to four major factors. First, the detrimental cross-talk between the acutely injured kidney and other organs implicates AKI as an instigator and multiplier of pulmonary, cardiac, hepatic, and neurologic dysfunction, which likely accounts for the vicious cycle of AKI and multi-organ failure leading to mortality [34]. Second, AKI impairs the immune function and markedly escalates susceptibility to infection [35]. This sets up another vicious cycle whereby infections lead to sepsis and AKI, and sepsis-induced immune dysfunction is further exacerbated by the AKI-induced immune dysregulation. Third, AKI contributes to medication failure from many reasons. On the one hand, critically ill children with AKI are at substantial risk for adverse outcomes of drug therapy, due to reduced renal clearance, decrease in protein binding, and decrease in drug metabolism [36-38]. On the other hand, under-dosing of critical medications such as antibiotics often occurs, due to unstable elimination rates 
Table 2 Novel urinary biomarkers for the prediction of AKI and its outcomes

\begin{tabular}{|c|c|c|c|c|c|}
\hline Biomarker & Source & Function & Cardiac surgery & Kidney transplant & ICU/ED \\
\hline NGAL & $\begin{array}{l}\text { Distal tubule } \\
\text { and } \\
\text { collecting } \\
\text { duct }\end{array}$ & $\begin{array}{l}\text { Regulates iron } \\
\text { trafficking, promotes } \\
\text { tubule cell survival }\end{array}$ & $\begin{array}{l}2 \mathrm{~h} \text { post CPB } 2 \text { days pre AKI } \\
\text { Predicts AKI severity, } \\
\text { dialysis, and death }\end{array}$ & $\begin{array}{l}6 \mathrm{~h} \text { post transplant } \\
2-3 \text { days pre DGF } \\
\text { Predicts long-term graft } \\
\text { loss }\end{array}$ & $\begin{array}{l}\text { On admission 1-2 days pre } \\
\text { AKI Predicts AKI severity, } \\
\text { dialysis, and death }\end{array}$ \\
\hline IL-18 & $\begin{array}{l}\text { Proximal } \\
\text { tubule }\end{array}$ & $\begin{array}{l}\text { Promotes tubule cell } \\
\text { apoptosis and } \\
\text { necrosis }\end{array}$ & $\begin{array}{l}6 \text { h post CPB } 2 \text { days pre AKI } \\
\text { Predicts AKI severity, } \\
\text { dialysis, and death }\end{array}$ & $\begin{array}{l}6 \text { h post transplant } \\
2-3 \text { days pre DGF } \\
\text { Predicts long-term graft } \\
\text { loss }\end{array}$ & $\begin{array}{l}\text { On admission 1-2 days pre } \\
\text { AKI Predicts AKI severity, } \\
\text { dialysis, and death }\end{array}$ \\
\hline L-FABP & $\begin{array}{l}\text { Proximal } \\
\text { tubule }\end{array}$ & $\begin{array}{l}\text { Antioxidant, } \\
\text { suppresses tubule- } \\
\text { interstitial damage }\end{array}$ & $\begin{array}{l}6 \text { h post CPB } 2 \text { days pre AKI } \\
\text { Not tested for outcomes }\end{array}$ & Not tested & $\begin{array}{l}\text { On admission 1-2 days pre } \\
\text { AKI Predicts AKI severity, } \\
\text { dialysis, and death }\end{array}$ \\
\hline KIM-1 & $\begin{array}{l}\text { Proximal } \\
\text { tubule }\end{array}$ & $\begin{array}{l}\text { Promotes epithelial } \\
\text { regeneration, } \\
\text { regulates apoptosis }\end{array}$ & $\begin{array}{l}12 \mathrm{~h} \text { post CPB } 1 \text { day pre AKI } \\
\text { Not tested for outcomes }\end{array}$ & Not tested & $\begin{array}{l}\text { On admission } 1-2 \text { days pre } \\
\text { AKI Predicts AKI severity, } \\
\text { dialysis, and death }\end{array}$ \\
\hline
\end{tabular}

Times shown (in hours or days) are the earliest time points when the biomarker becomes significantly increased from baseline

$A K I$ acute kidney injury, defined as AKIN Stage I or greater, $C P B$ cardiopulmonary bypass, $D G F$ delayed graft function, $I C U$ intensive care unit, $E D$ emergency department, $I L-18$ interleukin-18, KIM-1 kidney injury molecule 1, L-FABP liver-type fatty acid binding protein, $N G A L$ neutrophil gelatinase-associated lipocalin

and volumes of distribution, and significant clearance by renal replacement therapies [36-38].

Fourth, and perhaps most importantly, AKI often results in fluid overload, and fluid overload is a common accompaniment to several clinical situations (e.g., sepsis, hypotension, cardiac surgery) that lead to AKI. Pediatric studies have pioneered the concept, now well established in all ages, that fluid overload is an independent risk factor for mortality in AKI. A recent analysis of 340 children used a tripartite classification for percent fluid overload at initiation of renal replacement therapy [39••]: $<10 \%, 10-20 \%$, and $\geq 20 \%$ fluid overload. Those with $\geq 20 \%$ fluid overload had a $66 \%$ mortality rate, whereas those with 10-20\% fluid overload displayed a lower mortality rate of $43 \%$, and those with $<10 \%$ fluid overload had the lowest mortality rate of $29 \%$. The association between degree of fluid overload and mortality remained after adjusting for intergroup differences and severity of illness [39••]. Patients with $\geq 20 \%$ fluid overload had an 8.5 -fold greater adjusted odds ratio of death than those with $<20 \%$ fluid overload. Preventing fluid overload and rapid correction of fluid overload with early initiation of renal replacement therapy may represent "low-hanging" fruits in pediatric AKI therapeutics that deserve investigation.

\section{Can Pediatric AKI Risk be Predicted using Clinical Measures?}

AKI lacks physical symptoms and signs in the early stages when interventions are likely to be most effective. Investigators have recently sought to integrate contextual risk factors with evidence of renal injury to stratify AKI risk
$[40 \bullet, 41]$. Termed "renal angina", a three-tiered schema empirically places children into moderate (any ICU admission plus doubling of serum creatinine or fluid overload $>15 \%$ ), high (heart failure or stem cell transplant plus serum creatinine increase $\geq 0.3 \mathrm{mg} / \mathrm{dl}$ or fluid overload $>10 \%$ ), and very high risk for AKI (mechanical ventilation and vasoactive medication plus any increase in serum creatinine or fluid overload $>5 \%$ ). Thus, as the AKI risk increases (e.g., mechanical ventilation), less evidence of AKI is needed (e.g., small changes in serum creatinine) to meet the threshold for renal angina. In analogy with cardiac angina, the major goal of renal angina determination is to identify children who will maximally benefit from biomarker measurement for prediction and early treatment of AKI. Studies to validate the utility of renal angina are currently in progress.

\section{Can Pediatric AKI and its Outcomes be Predicted Using Biomarkers?}

The genomic and proteomic tools of modern science have identified novel markers for the early stress response of the kidney to AKI, which are induced in the kidney tubules during the early damage phase, and serendipitously appear in the urine or plasma well before a change in serum creatinine is detected $[2,5]$. Many are being developed and validated as early non-invasive biomarkers for the prediction of AKI and its clinical outcomes in humans. This is a rapidly evolving field, and the current status of the most promising examples is shown in Table 2.

The most widely studied and validated early biomarker of AKI in children is neutrophil gelatinase-associated 
lipocalin (NGAL). In prospective studies of children undergoing cardiopulmonary bypass, levels of NGAL in the urine and plasma were significantly elevated within 2-6 $\mathrm{h}$ of bypass in those who subsequently developed AKI $[42-45,46 \bullet \bullet, 47 \bullet \cdot]$. Strong associations between early NGAL measurements and hard clinical outcomes, including length of hospital stay and the duration and severity of pediatric AKI, have now been documented [43-45, 46••, 47•.0]. Furthermore, the additional of NGAL significantly improves the risk prediction for AKI after cardiac surgery over clinical models alone [47••]. Studies in the more heterogeneous pediatric intensive care $[48,49]$ and pediatric emergency department settings [50] also demonstrated that NGAL predicted AKI about 1-2 days prior to the rise in serum creatinine, with high sensitivity. Two large multicenter pooled analyses of existing NGAL studies in children and adults have recently been published, confirming the utility of this marker for the early diagnosis of AKI and its clinical outcomes [51, 52••].

A recent study examined a combination of biomarkers in 220 children undergoing cardiac surgery [46••]. Urinary NGAL was increased in AKI patients within $2 \mathrm{~h}$ of bypass initiation, urine IL-18 and L-FABP were increased within $6 \mathrm{~h}$, and urine KIM-1 increased at the $12 \mathrm{~h}$ time point. All markers correlated with AKI severity and clinical outcomes, and improved the risk prediction for AKI over clinical models. Thus, they represent temporally sequential markers, and a panel of such biomarkers may therefore help establish the timing of injury and plan appropriate therapies [46••]. Standardized clinical laboratory platforms for the measurement of urine [44] and plasma [43] NGAL are now available in most countries.

It is anticipated that biomarkers of early structural AKI such as NGAL will provide critical diagnostic and prognostic stratification, independent of functional markers such as serum creatinine. While biomarker combinations may be necessary to provide the best information in a context-specific manner, the technical and financial challenges of developing biomarker panels are substantial. It is therefore vital that large enough future studies demonstrate (a) the association between early structural biomarkers and hard outcomes such as dialysis, cardiovascular events, and death, both with and independent of functional markers, and (b) that randomization to a treatment for AKI based on high structural biomarker levels results in an improvement in kidney function and amelioration of adverse clinical outcomes.

\section{Conclusion}

The Kidney Disease: Improving Global Outcomes (KDIGO) group has provided a consensus definition and classification for pediatric AKI, the uniform adoption of which will improve our understanding of pediatric AKI epidemiology and raise awareness of this increasingly common condition. Pediatric AKI leads to CKD and ESRD, but we need to identify clinical risk factors and novel biomarkers that predict these outcomes. A concerted effort is needed to strengthen the emerging recommendation that children with AKI require long-term evaluation for CKD. Preventing fluid overload and rapid correction of fluid overload with early initiation of renal replacement therapy represent pediatric AKI therapeutics that deserves urgent investigation. Excellent point-of-care early biomarkers of AKI have now become available. Biomarkerguided therapies, based on "renal angina" scoring, should be the next major challenge to overcome, in order to dramatically improve the outcome of pediatric AKI.

Conflicts of interest No potential conflicts of interest relevant to this article were reported.

\section{References}

Papers of particular interest, published recently, have been highlighted as:

- Of importance

•• Of major importance

1. • Kidney Disease. Improving global outcomes (KDIGO) clinical practice guideline for acute kidney injury. Kidney Int Suppl. 2012;2:19-36. These are the most recent recommendations for the definition and staging of $A K I$

2. Devarajan P. Neutrophil gelatinase-associated lipocalin: a promising biomarker for human acute kidney injury. Biomarkers Med. 2010;4(2):265-80.

3. Askenazi D. Evaluation and management of critically ill children with acute kidney injury. Curr Opin Pediatr. 2011;23(2):201-7.

4. - Zappitelli M, Bernier P-L, Saczkowski RS, et al. A small postoperative rise in serum creatinine predicts acute kidney injury in children undergoing cardiac surgery. Kidney Int. 2009;76:885-92. This paper highlights the prognostic significance of even a small change in serum creatinine in children after cardiac surgery

5. Devarajan P. Biomarkers for the early detection of acute kidney injury. Curr Opin Pediatr. 2011;23(2):194-200.

6. Goldstein SL, Devarajan P. Acute kidney injury in childhood: should we be worried about progression to CKD? Pediatr Nephrol. 2011;26(4):509-22.

7. Goldstein SL, Devarajan P. Acute kidney injury leads to pediatric patient mortality. Nat Rev Nephrol. 2010;6:393-4.

8. Askenazi DJ. Do children with acute kidney injury require longterm evaluation for CKD? Am J Kidney Dis. 2012;59(4):478-80.

9. Palmieri T, Lavrentieva A, Greenhlagh D. An assessment of acute kidney injury with modified RIFLE criteria in pediatric patients with severe burns. Intensive Care Med. 2009;35:2125-9.

10. Duzova A, Bakkaloglu A, Kalyoncu M, et al. Etiology and outcome of acute kidney injury in children. Pediatr Nephrol. 2010; 25:1453-61.

11. Zappitelli M, Moffett BS, Hyder A, et al. Acute kidney injury in non-critically ill children treated with aminoglycoside antibiotics 
in a tertiary healthcare centre: a retrospective cohort study. Nephrol Dial Transpl. 2011;26:144-50.

12. Slater MB, Anand V, Uleryk EM, et al. A systematic review of RIFLE criteria in children, and its application and association with measures of mortality and morbidity. Kidney Int. 2012;81: 791-8.

13. $\bullet$ Shneider J, Khemani R, Grushkin C, et al. Serum creatinine as stratified in the RIFLE score for acute kidney injury is associated with mortality and length of stay for children in the pediatric intensive care unit. Crit Care Med. 2010;38:933-9. This is the largest study reported to date, illustrating the incidence and prognosis of AKI in critically ill children

14. Alkandari O, Eddington KA, Hyder A, et al. Acute kidney injury is an independent risk factor for pediatric intensive care unit mortality, longer length of stay and prolonged mechanical ventilation in critically ill children: a two-center retrospective cohort study. Crit Care. 2011;15(3):R146.

15. Kavaz A, Ozcakar ZB, Kendirli T, et al. Acute kidney injury in a paediatric intensive care unit: comparison of the pRIFLE and AKIN criteria. Acta Paediatr. 2012;101(3):e126-9.

16. Prodhan P, McCage LS, Stroud MH, et al. Acute kidney injury is associated with increased in-hospital mortality in mechanically ventilated children with trauma. J Trauma Acute Care Surg. 2012;73(4):832-7.

17. Krawczeski CD, Woo JG, Wang Y, et al. Neutrophil gelatinaseassociated lipocalin concentrations predict development of acute kidney injury in neonates and children after cardiopulmonary bypass. J Pediatr. 2011;158:1009-15.

18. Fadel FI, Abdel Rahman AMO, Mohamed MF, et al. Plasma neutrophil gelatinase-associated lipocalin as an early biomarker for prediction of acute kidney injury after cardio-pulmonary bypass in pediatric cardiac surgery. Arch Med Sci. 2012;8(2):250-5.

19. Li S, Krawczeski CD, Zappitelli M, et al. Incidence, risk factors, and outcomes of acute kidney injury after pediatric cardiac surgery: a prospective multicenter study. Crit Care Med. 2011;39:1493-9.

20. Blinder JJ, Goldstein SL, Lee V-V, et al. Congenital heart surgery in infants: effects of acute kidney injury on outcomes. J Thorac Cardiovasc Surg. 2012;143:368-74.

21. Toth R, Breuer T, Cserep Z, et al. Acute kidney injury is associated with higher morbidity and resource utilization in pediatric patients undergoing heart surgery. Ann Thorac Surg. 2012; 93(6):1984-90.

22. Aydin SI, Seiden HS, Blaufox AD, et al. Acute kidney injury after surgery for congenital heart disease. Ann Thorac Surg. 2012. doi:10.1016/j.athoracsur.2012.06.050

23. Moffett BS, Goldstein SL. Acute kidney injury and increasing nephrotoxic-medication exposure in noncritically-ill children. Clin J Am Soc Nephrol. 2011;6(4):856-63.

24. Kellum JA, Bellomo R, Ronco C. Kidney attack. JAMA. 2012;307(21):2265-6.

25. Bresolin N, Silva C, Hallal A, et al. Prognosis for children with acute kidney injury in the intensive care unit. Pediatr Nephrol. 2009;24(3):537-44.

26. Symons JM, Chua AN, Somers MJ, et al. Demographic characteristics of pediatric continuous renal replacement therapy registry. Clin J Am Soc Nephrol. 2007;2:732-8.

27. Coca SG, Singanamala S, Parikh CR. Chronic kidney disease after acute kidney injury: a systematic review and meta-analysis. Kidney Int. 2012;81(5):442-8.

28. Garg AX, Suri RS, Barrowman N, et al. Long-term renal prognosis of diarrhea-associated hemolytic uremic syndrome: a systematic review, meta-analysis, and meta-regression. JAMA. 2003;290:1360-70.

29. Askenazi DJ, Feig DI, Graham NM, et al. 3-5 year longitudinal follow-up of pediatric patients after acute renal failure. Kidney Int. 2006;69:184-9.
30. - Mammen C, Al Abbas A, Skippen P, et al. Long-term risk of CKD in children surviving episodes of acute kidney injury in the intensive care unit: a prospective cohort study. Am J Kidney Dis. 2012;59(4):523-30. This manuscript demonstrates the long-term sequelae of AKI in children

31. Go AS, Parikh CR, Ikizler TA, et al. The assessment, serial evaluation, and subsequent sequelae of acute kidney injury (ASSESS-AKI) study: design and methods. BMC Nephrol. 2010;27(11):22.

32. Basile DP, Friedrich JL, Spahic J, et al. Impaired endothelial proliferation and mesenchymal transition contribute to vascular rarefaction following acute kidney injury. Am J Physiol Ren Physiol. 2011;300:F721-33.

33. Ko GJ, Grigoryev DN, Linfert D, et al. Transcriptional analysis of kidneys during repair from AKI reveals possible roles for NGAL and KIM-1 as biomarkers of AKI-to-CKD transition. Am J Physiol Ren Physiol. 2010;298:F1472-83.

34. Grams ME, Rabb H. The distant organ effects of acute kidney injury. Kidney Int. 2012;81(10):942-8.

35. Singbartl K, Bishop JV, Wen X, et al. Differential effects of kidney-lung cross-talk during acute kidney injury and bacterial pneumonia. Kidney Int. 2011;80(6):633-44.

36. Awdishu L, Bouchard J. How to optimize drug delivery in renal replacement therapy. Semin Dial. 2011;24(2):176-82.

37. Eyler RF. Medscape. Antibiotic dosing in critically ill patients with acute kidney injury. Nat Rev Nephrol. 2011;7(4):226-35.

38. Perazella MA. Drug use and nephrotoxicity in the intensive care unit. Kidney Int. 2012;81(12):1172-8.

39. •- Sutherland SM, Zappitelli M, Alexander SR, et al. Fluid overload and mortality in children receiving continuous renal replacement therapy: the prospective pediatric continuous renal replacement therapy registry. Am J Kidney Dis. 2010;55:316-25. This manuscript illustrates the close relationship between fluid overload and mortality in children

40. - Basu RK, Chawla LS, Wheeler DS, et al. Renal angina: an emerging paradigm to identify children at risk for acute kidney injury. Pediatr Nephrol. 2012;27:1067-78. This manuscript introduces the concept of "renal angina" as a tool to identify children who will maximally benefit from AKI biomarker measurements

41. Goldstein SL, Chawla LS. Renal angina. Clin J Am Soc Nephrol. 2010;5:943-9.

42. Mishra J, Dent C, Tarabishi R, et al. Neutrophil gelatinaseassociated lipocalin (NGAL) as a biomarker for acute renal injury after cardiac surgery. Lancet. 2005;365:1231-8.

43. Dent CL, Ma Q, Dastrala S, et al. Plasma neutrophil gelatinaseassociated lipocalin predicts acute kidney injury, morbidity and mortality after pediatric cardiac surgery: a prospective uncontrolled cohort study. Crit Care. 2007;11:R127.

44. Bennett M, Dent CL, Ma Q, et al. Urine NGAL predicts severity of acute kidney injury after cardiac surgery: a prospective study. Clin J Am Soc Nephrol. 2008;3:665-73.

45. Krawczeski CD, Woo JG, Wang Y, et al. Neutrophil gelatinaseassociated lipocalin concentrations predict development of acute kidney injury in neonates and children after cardiopulmonary bypass. J Pediatr. 2011;158:1009-15.

46. • Krawczeski CD, Goldstein SL, Woo JG, et al. Temporal relationship and predictive value of urinary acute kidney injury biomarkers after pediatric cardiopulmonary bypass. J Am Coll Cardiol. 2011;58:2301-9. This prospective study illustrates the temporal sequence of multiple biomarkers for the prediction of AKI after pediatric cardiac surgery

47. •• Parikh CR, Devarajan P, Zappitelli M, et al. Postoperative biomarkers predict acute kidney injury and poor outcomes after pediatric cardiac surgery. J Am Soc Nephrol. 2011;22:1737-47. This prospective multicenter demonstrates the value of biomarkers 
for the prediction of adverse outcomes after pediatric cardiac surgery

48. Zappitelli M, Washburn KK, Arikan AA, et al. Urine NGAL is an early marker of acute kidney injury in critically ill children. Crit Care. 2007;11(4):R84

49. Wheeler DS, Devarajan P, Ma Q, et al. Serum neutrophil gelatinase-associated lipocalin (NGAL) as a marker of acute kidney injury in critically ill children with septic shock. Crit Care Med. 2008;36(4):1297-303.

50. Du Y, Zappitelli M, Mian A, et al. Urinary biomarkers to detect acute kidney injury in the pediatric emergency center. Pediatr Nephrol. 2011;26(2):267-74.
51. Haase M, Bellomo R, Devarajan P, et al. Accuracy of neutrophil gelatinase-associated lipocalin (NGAL) in diagnosis and prognosis in acute kidney injury: a systematic review and metaanalysis. Am J Kidney Dis. 2009;54:1012-24. This multicenter pooled analysis of prospective studies illustrates the ability of $N G A L$ to predict adverse outcomes independent of serum creatinine

52. • Haase M, Devarajan P, Haase-Fielitz A, et al. The outcome of neutrophil gelatinase-associated lipocalin-positive subclinical acute kidney injury: a multicenter pooled analysis of prospective studies. J Am Coll Cardiol. 2011;57:1752-61. 